\title{
Spin Torque in Semiconductor Single Planar Tunnel Junctions
}

\author{
M. WilCZYŃSKI ${ }^{a}$, J. BARNAŚ ${ }^{b, c}$ AND R. ŚWIRKOWICZ ${ }^{a}$ \\ ${ }^{a}$ Faculty of Physics, Warsaw University of Technology \\ Koszykowa 75, 00-662 Warsaw, Poland \\ ${ }^{b}$ Department of Physics, Adam Mickiewicz University \\ Umultowska 85, 61-614 Poznań, Poland \\ ${ }^{c}$ Institute of Molecular Physics, Polish Academy of Sciences \\ Smoluchowskiego 17, 60-179 Poznań, Poland

\begin{abstract}
Transport in a single planar tunnel junction with electrodes made of a ferromagnetic semiconductor is analyzed theoretically in the zero-temperature limit. Tunneling current and both (in-plane and out-of-plane) components of the spin torque exerted on one of the ferromagnetic electrodes are determined as a function of the angle $\theta$ between magnetic moments of the electrodes. The influence of the bias voltage and spin splitting of the electron band (in both electrodes) on the spin torque components is analyzed numerically.
\end{abstract}

PACS numbers: 73.40.Gk, 73.40.Ty

\section{Introduction}

Electrons tunneling through a barrier between two ferromagnetic electrodes can exert a torque on the magnetic moments of both electrodes. This torque (called a spin torque) is a consequence of the spin transfer from conduction electrons to the localized magnetic moments [1]. When the spin torque is sufficiently large, it can lead to switching of the magnetic moments from parallel to antiparallel alignment, or vice versa, depending on the direction of tunneling current. Experiments confirming the magnetic switching have been recently performed in metallic [2] as well as semiconductor [3] tunnel junctions. In the latter case the current needed to rotate the magnetic moments is usually lower than that in metallic junctions [3]. These experiments have also stimulated further experimental and theoretical works (see e.g. [4]) on the current induced magnetic switching (CIMS). It is worth noting that the CIMS phenomenon is promising for various applications, e.g. in magnetic memory elements. 
The spin torque has generally two components: the in-plane and out-of-plane ones. The in-plane component is oriented in the plane determined by the magnetic moments of both electrodes, while the out-of-plane component is normal to this plane (therefore this component is also called normal).

\section{Model}

In this paper we consider a single planar tunnel junction with two ferromagnetic semiconductor layers separated by a nonmagnetic barrier (see inset in Fig. 1c). Both magnetic layers are assumed to be thick enough to be considered as semi-infinite electrodes. We also assume that for positive bias voltage electrons tunnel from the left to the right electrode. The associated charge current is then defined as positive (and flows from right to left). The in-plane $T_{\|}$and out-of-plane $T_{\perp}$ components of the spin torque exerted on the right ferromagnetic electrode are calculated and analyzed as a function of the angle $\theta$ between magnetic moments of the electrodes, spin-splitting of the electron band in both electrodes, and bias voltage applied to the system.

The calculations have been performed in the zero-temperature limit and in the free-electron-like one-band model. Accordingly, the electronic structure of ferromagnetic electrodes is modeled by a parabolic spin-split band with the free electron mass [5]. The arbitrary angle $\theta$ between the net spin moments of the left $S^{\mathrm{l}}$ and right $S^{\mathrm{r}}$ electrode is taken into account in the relevant matching conditions for the electron wave function [1]. It is assumed that the electrodes' magnetic moments are oriented in the junction plane, so the normal torque is perpendicular to the electrode/barrier interfaces, whereas in-plane torque is oriented in the junction plane. The spin torque components have been determined from the conservation of the total angular momentum, i.e. momentum of localized moments and that carried by spin current associated with charge current. In the junctions with thick right electrode the in-plane (normal) torque can be calculated simply as

$$
T_{\|(\perp)}=\frac{\hbar}{2} J_{x^{\prime}(y)}
$$

The spin current components $J_{x^{\prime}}$ and $J_{y}$ are taken in the barrier at the interface with the right electrode and in the local coordinate system of the right electrode. The $\mu$-th component $\left(\mu=x^{\prime}, y\right)$ of the spin current corresponding to electrons described by the wave function $\Psi(y)$ is calculated from the general expression

$$
j_{\mu}^{s}(y)=\frac{\mathrm{i} \hbar}{2 m}\left[\left(\frac{\partial}{\partial y} \Psi^{+}(y)\right) \sigma_{\mu} \Psi(y)-\Psi^{+}(y) \sigma_{\mu} \frac{\partial}{\partial y} \Psi(y)\right],
$$

where $\sigma_{\mu}$ are the Pauli matrices, whereas $\Psi(y)$ has the spinor form in the relevant local reference frame. To find the total spin current $J_{x^{\prime}}$ and $J_{y}$, one has to sum contributions from electrons of different in-plane wave vectors and energies from the range between the Fermi levels in both electrodes [5]. 


\section{Results and discussion}

Numerical calculations have been performed for the parameters corresponding to systems with semiconductor ferromagnetic electrodes: the Fermi energy $E_{\mathrm{f}}=0.1 \mathrm{eV}$, the barrier height $U=0.1 \mathrm{eV}$, and the barrier thickness $d=2.0 \mathrm{~nm}$. The results presented in Fig. 1 were obtained for $\theta=\pi / 2$. From the inset in Fig. 1a it follows that the in-plane and normal spin torque components are maximal for $\theta=\pi / 2$ and vanish in the collinear configurations. Additionally, the normal torque is smaller than the in-plane one, however it cannot be neglected. In Figs. 1a and b both components of spin torque divided by the corresponding charge currents are shown as a function of the spin splitting of the electron bands
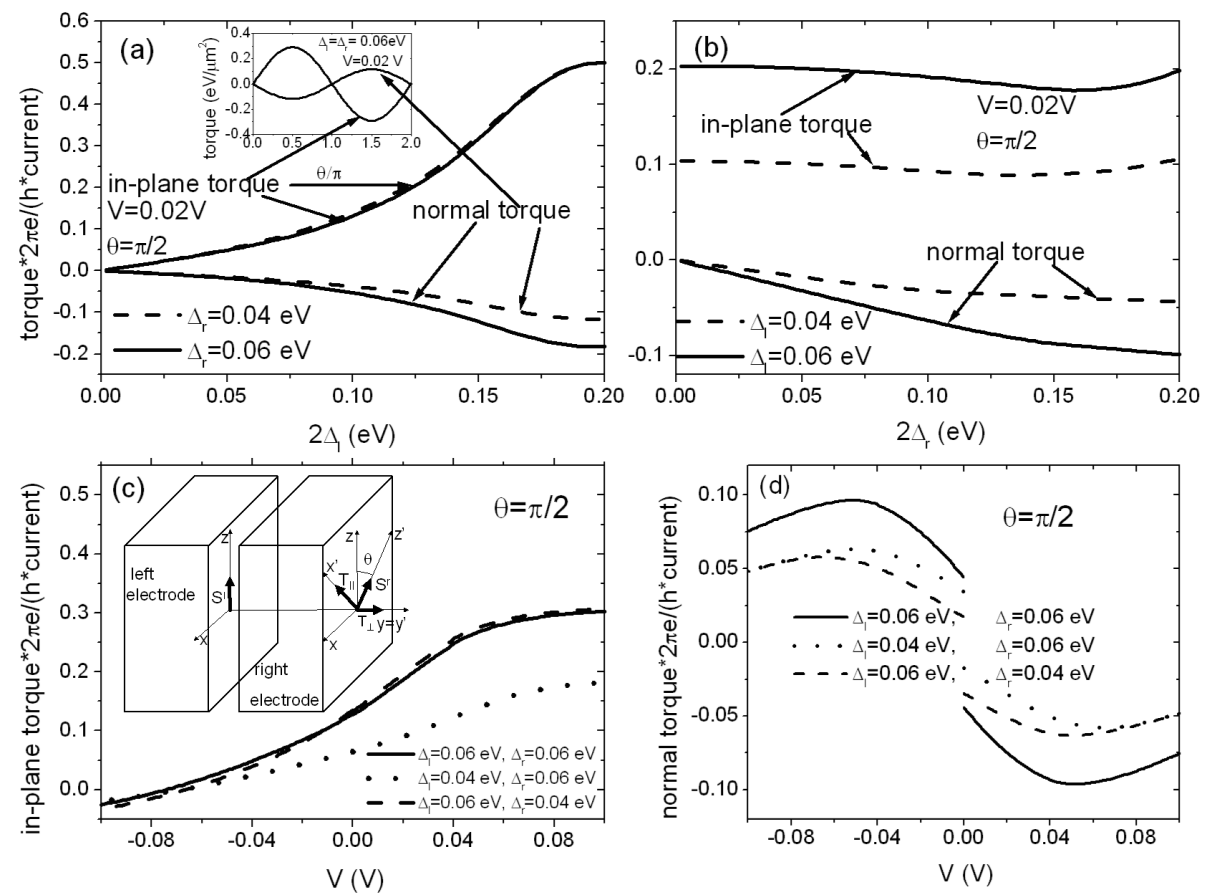

Fig. 1. The in-plane and normal spin torque components divided by the charge current, calculated as a function of the spin splitting of electron bands in the left $2 \Delta_{1}$ (a) and right $2 \Delta_{\mathrm{r}}$ (b) electrodes and the bias voltage (c), (d) applied to the junction (the charge current is negative for $V<0$ ). The inset in Fig. 1a shows the $\theta$ dependence of both torque components, whereas the inset in Fig. 1c shows the geometry of the system.

in the left, $2 \Delta_{\mathrm{l}}$ (Fig. 1a) and right, $2 \Delta_{\mathrm{r}}$ (Fig. 1b) electrodes for the bias voltage $V=0.02 \mathrm{~V}$ and for different values of the spin splitting in the other electrode. One can see that the normalized in-plane torque exerted on the right electrode increases significantly with increasing $\Delta_{\mathrm{l}}$, whereas it practically does not change with increasing $\Delta_{\mathrm{r}}$. It does not vanish even in junctions with $\Delta_{\mathrm{r}} \rightarrow 0$. Thus, this 
component of spin-torque can be significant even in junctions with very small spin polarization of the right electrode, provided that the polarization of the electrons in the left electrode is sufficiently large. This is because the in-plane normalized torque depends mainly on the effective spin polarization of the left electrode, as predicted by Slonczewski [1]. A different situation appears for the normal component of the spin torque. Its magnitude increases with increasing $\Delta_{\mathrm{l}}$ and $\Delta_{\mathrm{r}}$, and tends to zero when $\Delta_{\mathrm{l}}$ or $\Delta_{\mathrm{r}}$ vanishes.

The bias dependence of the normalized in-plane torque, presented in Fig. 1c, is almost insensitive to the changes of $\Delta_{\mathrm{r}}$, whereas the similar curves calculated for the normal torque (Fig. 1d) depend on $\Delta_{\mathrm{r}}$. As one can see the normalized in-plane torque is clearly asymmetric with respect to the bias reversal, even in junctions with identical electrodes $\left(\Delta_{\mathrm{l}}=\Delta_{\mathrm{r}}\right)$. It increases with increasing positive bias voltage for $|e V| \leq U$, however this increase is rather weak for $|e V| \approx U$. For negative bias voltages, the in-plane torque decreases with increasing bias voltage and changes sign for a sufficiently large bias. On the other hand, the bias dependence of the magnitude of the normal component of the torque in junctions with $\Delta_{1}=\Delta_{\mathrm{r}}$ is symmetric with respect to the bias reversal. It is non-symmetric in junctions with $\Delta_{\mathrm{l}} \neq \Delta_{\mathrm{r}}$, but the asymmetry is less pronounced than in the case of the in-plane torque.

\section{References}

[1] J.C. Slonczewski, Phys. Rev. B 71, 024411 (2005); 39, 6995 (1989).

[2] Y. Huai, F. Albert, P. Nguyen, M. Pakala, T. Valet, Appl. Phys. Lett. 84, 3118 (2004).

[3] D. Chiba, Y. Sato, T. Kita, F. Matsukura, H. Ohno, Phys. Rev. Lett. 93, 216602 (2004); M. Elsen, O. Boulle, J.-M. George, H. Jaffrès, R. Mattana, V. Cros, A. Fert, A. Lemaitre, R. Giraud, G. Faini, Phys. Rev. B 73, 035303 (2006).

[4] I. Theodonis, N. Kioussis, A. Kalitsov, M. Chshiev, W.H. Butler, Phys. Rev. Lett. 97, 237205 (2006).

[5] M. Wilczyński, J. Barnaś, Acta Phys. Pol. A 97, 443 (2000). 